\title{
PROPUESTA DE UNA METODOLOGÍA FORMAL PARA EL DISEÑO DE SISTEMAS DE CONTROL CINEMÁTICO Y DINÁMICO EN MANIPULADORES INDUSTRIALES SERIALES
}

\author{
PROPOSAL OF A FORMAL METHODOLOGY FOR THE DESIGN OF \\ KINEMATIC AND DYNAMIC CONTROL SYSTEMS IN SERIAL INDUSTRIAL \\ HANDLERS
}

\author{
Ing. María Carolina Duque Suárez, Ing. Diana Marcela Rodríguez Rodríguez. \\ Esp. Sergio Iván Quintero Ayala, MSc. Oscar Manuel Duque Suárez.
}

Universidad Francisco de Paula Santander, Facultad de Ingeniería, Grupo "GIAC". Ave. Gran Colombia, No. 12E-96, San José de Cúcuta, Norte de Santander, Colombia.

Tel.: (+577) 577 6655, Fax: (+577) 5753893.

E-mail: \{carolinaduke, dimaro4\}@ @otmail.com

\begin{abstract}
Resumen: En este trabajo se realiza un planteamiento metodológico para el diseño de un sistema de control cinemático y dinámico en manipuladores seriales a través del análisis de simulación, lo cual permite observar el comportamiento de estos sistemas de control con respecto al planteamiento y tomar decisiones con respecto al diseño del manipulador.
\end{abstract}

Palabras clave: Control cinemático, control dinámico, manipulador serial industrial, articulaciones, trayectorias, interpolador.

\begin{abstract}
This works deals with a methodological approach for the design of a system of kinematic and dynamic control in serial manipulators through simulation analysis is performed, which allows observing the behavior of these control systems in approach and make decisions with respect the design of the manipulator.
\end{abstract}

Keywords: Kinematic control, dynamic control, industrial serial manipulator, joints, paths, interpolator.

\section{INTRODUCCIÓN}

En la actualidad los robots industriales tienen un lugar destacado dentro de la automatización ya que vivimos en un mundo globalizado que genera alta competitividad industrial y estándares de producción cada vez más elevados, además de tasas de producción más altas, eficientes y flexibles; es en este contexto donde la robótica de manipuladores ha tenido un gran auge (Cieza, 2011).

Una de las últimas aplicaciones en los manipuladores industriales ha sido los robots de búsqueda y rescate, que con la ayuda de visión artificial, controlada a partir de algoritmos desarrollados que incluyen la planificación para la manipulación de robots en presencia de obstáculos móviles. Este se analiza mediante un algoritmo exacto que se alcanza normalmente en bajo tiempo de funcionamiento o de un algoritmo que alcanza normalmente bajo error (Kris Hauser, 2014).

El control de robots manipuladores tiene muchas más posibilidades de continuar desarrollándose, además los beneficios que trae consigo, permite el progreso de diversas áreas para las cuales puede ser aplicada la robótica como es el caso de la medicina, la industria, la exploración y situaciones riesgosas para el ser humano (Cieza, 2011). 
El análisis desde el punto de vista mecánico de un robot se puede efectuar atendiendo exclusivamente a sus movimientos (cinemática) o atendiendo además a las fuerzas y momentos que actúan sobre sus partes (dinámica) debidos a los elementos actuadores y a la carga transportada por el elemento terminal. Debido a esto, este documento orientado en realizar una metodología para el diseño del control cinemático y dinámico de un manipulador serial industrial, utilizando simulaciones en la herramienta computacional Matlab, para la comprobación de dicha metodología en un manipulador de 4GDL (Grados de libertad) con dos articulaciones prismáticas y dos rotacionales, demostrando los tipos de trayectorias, interpoladores y controles dinámicos aplicados a dicho manipulador.

\section{CONTROL CINEMÁTICO Y DINÁMICO}

El control cinemático establece cuales son las trayectorias que debe seguir cada articulación del robot a lo largo del tiempo para lograr los objetivos fijados por el usuario (punto de destino, trayectoria cartesiana del efecto final del robot, tiempo invertido por el usuario, etc.). Estas trayectorias se seleccionaran atendiendo a las restricciones físicas propias de los accionamientos y a ciertos criterios de calidad de trayectoria, como suavidad o precisión de la misma (Barrientos, 2007).

\subsection{Tipos de trayectorias}

Para realizar una tarea determinada el robot debe moverse desde un punto inicial a un punto final. Este movimiento puede ser realizado según infinitas trayectorias espaciales. De todas ellas hay algunas que, bien por su sencillez de implementación por parte del control cinemático o bien por su utilidad y aplicación a diversas tareas, son las que en la práctica incorporan los robots comerciales. De este modo, puede encontrarse que los robots dispongan de trayectorias punto a punto, coordinadas y continúas (Barrientos, 2007).

\subsubsection{Trayectorias punto a punto}

No importa el camino del extremo del robot. Sólo importa que alcance el punto final indicado (Barrientos, 2007).

Tipos:

- Movimiento eje a eje: Solo se mueve un eje cada vez (aumento del tiempo de ciclo). Solo en robots muy simples o con unidad de control limitada.
- Movimiento simultaneo de ejes: Los ejes inician su recorrido al mismo tiempo, y cada uno termina su trayectoria de manera independiente (altos requerimientos inútiles).

- Movimiento coordinado: Empiezan y terminan al mismo tiempo.

\subsubsection{Trayectorias coordinadas o isócronas}

No importa el camino del extremo del robot, pero los ejes se mueven simultáneamente, ralentizando las articulaciones más rápidas, de forma que todos los ejes terminan su recorrido a la vez (Barrientos, 2007).

- Tiempo total $=$ menor posible

- Se evitan exigencias inútiles de velocidad y aceleración

\subsubsection{Trayectorias continuas}

- Se pretende que el extremo del robot describa una trayectoria concreta y conocida.

- Importa el camino, pues durante el mismo el robot realiza parte de su cometido (soldadura por arco, corte por láser, etc.) (Barrientos, 2007).

- Trayectorias típicas: Línea recta, arco de círculo, otras.

\subsection{Tipos de interpoladores:}

- Interpoladores lineales

- Interpoladores a tramos:

- Polinomios cúbicos y quinticos (splines).

- Interpoladores cuadráticos a tramos (trapezoidales o ajuste parabólico).

- Otros interpoladores

Por lo general se utilizan funciones polinómicas cuyos coeficientes se ajustan según restricciones (Barrientos, 2007).

\subsection{Control dinámico}

El control dinámico tiene por misión procurar que las trayectorias realmente seguidas por el robot $q(t)$ sean lo más parecidas posibles a las propuestas por el control cinemático $\mathrm{q}_{1} \mathrm{~d}(t)$. Para ello hace uso del conocimiento del modelo dinámico del robot y de las herramientas de análisis y diseño aportadas por la Teoría del Servocontrol (representación interna, estado, estabilidad de Lyapunov, control PID, control adaptativo, etc). (Barrientos, 2007).

\subsubsection{Controlmonoarticular}


Las características mecánicas de un robot (dimensiones, peso, tipo de actuadores, etc.) influyen notablemente en el tipo de control a utilizar, por lo tanto se tienen en cuenta lo siguiente:

- Se considera cada articulación de forma independiente.

- No realista pero aceptable (perdida de prestaciones).

- La mayoría de los robots comerciales lo usan.

- Más aceptable en robots con alto factor de reducción.

\subsubsection{Control multiarticular}

\section{Características:}

En ocasiones la suposición de que el par $\tau_{p}$ (influencia del resto de las articulaciones sobre el control de una articulación) no es válida (accionamientos sin reductor, velocidades elevadas ) (Barrientos, 2007).

- Se debe entonces considerar al sistema como multivariable y acoplado.

- Elevada complejidad.

2.3.3 Control PID sobre el sistema desacoplado por prealimentación

- Las perturbaciones siempre están presentes, la prealimentación es insuficiente.

- No se conoce con exactitud D, H, C, K, Fv

- Utilizar un control PID, sobre el sistema desacoplado, para dar robustez al control frente a las incertidumbres anteriores.

- Al haber desacoplado al sistema, cada regulador PID será monovariable (Barrientos, 2007).

\subsubsection{Control Adaptativo}

- Control PID+FF $\rightarrow$ Eficiente si no hay perturbación y se conoce perfectamente el modelo.

- Los parámetros dinámicos no se conocen con presión o cambian.

- El control adaptativo permite adaptar el regular al cambio de los parámetros.

- Alternativamente se puede ajustar el regulador a unas condiciones intermedias de funcionamiento y asegurar que los movimientos del robot se mantienen estables en otras condiciones, aunque con su calidad dis minuida (Barrientos, 2007).

\section{METODOLOGÍA}

La metodología propuesta para el diseño de sistemas de control cinemático y dinámico en manipuladores industriales seriales establece una serie de pasos ordenados y concatenados para el desarrollo de los sistemas de control mencionados. En primer lugar se describió la metodología para el desarrollo de sistemas de control cinemático y sistemas de control dinámico en manipuladores industriales seriales y finalmente se unificaron las metodologías establecidas con la finalidad de determinar una única formal para el diseño de sistemas de control cinemático y dinámico en manipuladores industriales seriales.

\subsection{Metodología de control cinemático}

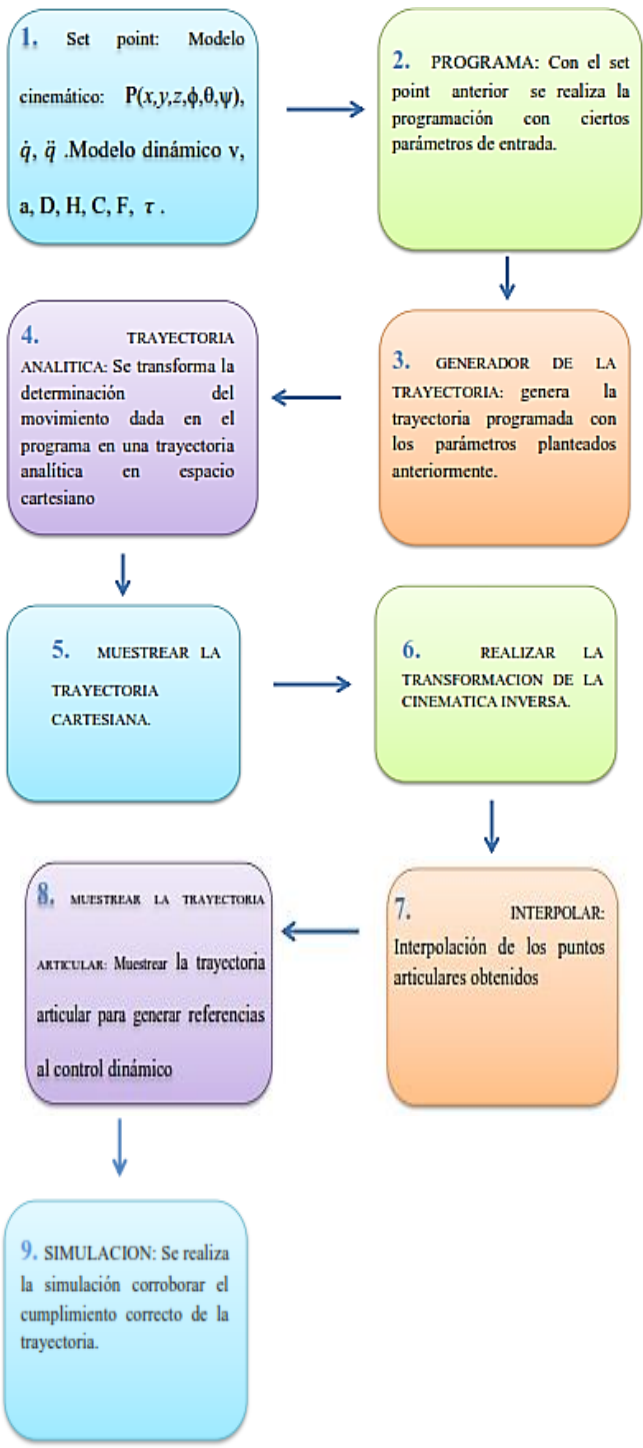

Fig. 1. Metodología del control cinemático.

\subsection{Metodología de control dinámico}




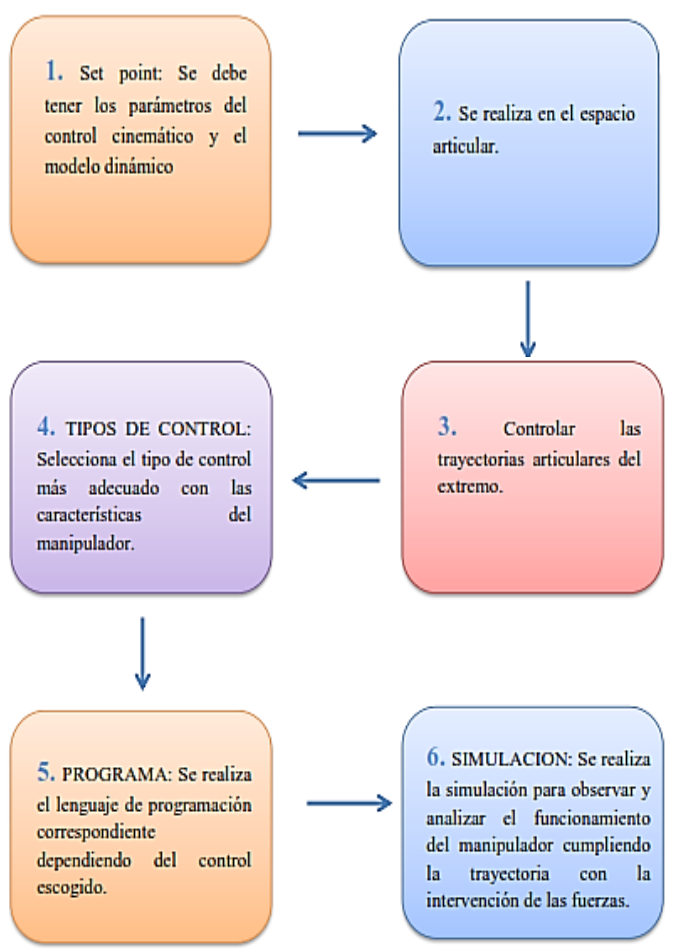

Fig. 2. Metodología del control cinemático.

\section{RESULTADOS - CASO DE ESTUDIO}

Los resultados obtenidos de las simulaciones realizadas al manipulador de 4GDL aplicándole la metodología propuesta anteriormente. En primer lugar se mostrará el control cinemático con algunas trayectorias e interpoladores y en segundo lugar se presentará el control dinámico.

\subsection{Control cinemático}

Para la comprensión de las simulaciones se crea una función que calcula la posición, velocidad y aceleración para $\mathrm{n}$ puntos de una trayectoria con un interpolador trapezoidal (velocidades inicial y final nulas).

Para el robot cilíndrico o manipulador de 4GDL su estructura se ensambló en Matlab y cada pieza fue creada en AutoCAD 2016 3D, este cumple los tres tipos de trayectorias obteniendo las gráficas de posición, aceleración y velocidad, seleccionando la trayectoria de movimiento coordinado las figuras 4, 5 son las representaciones de las posiciones en el espacio articular y el espacio de tarea, este movimiento permite un menor desgaste de las articulaciones al momento de realizar las trayectorias, las figuras 6,7 son las representaciones de la velocidad de cada articulación, las figuras 8, 9 muestran la aceleración de cada articulación, teniendo la velocidad y aceleración podemos observar que en el movimiento no se presenten cambios bruscos ya que pueden afectar el manipulador físicamente y no poder seguir la trayectoria programada.

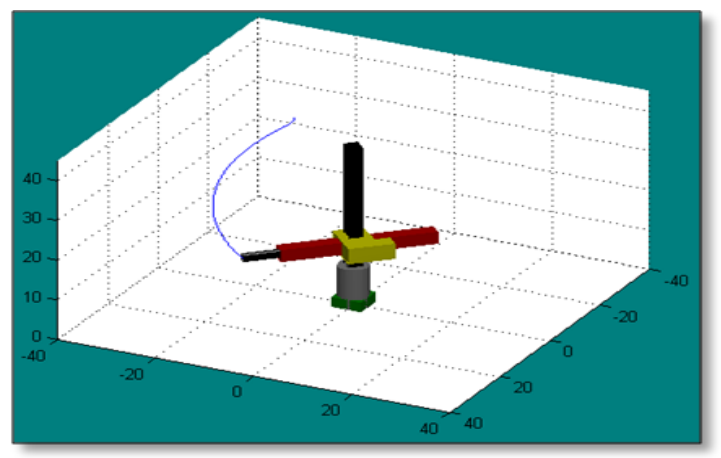

Fig. 3. Robot cilíndrico o robot de 4GDL cumple una trayectoria coordinada.

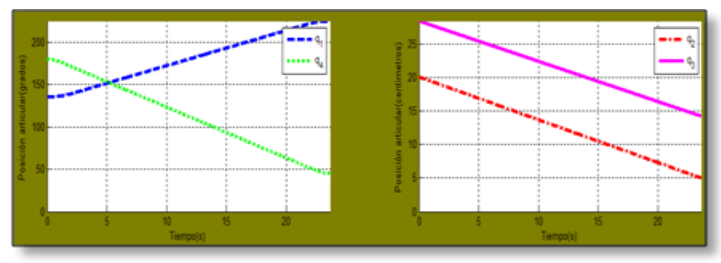

Fig. 4. Comportamiento la posición de cada articulación en el espacio articular trayectoria coordinada.

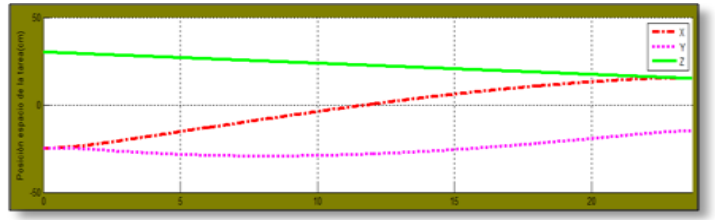

Fig. 5. Comportamiento la posición en el espacio de tarea en cada eje en trayectoria coordinada.

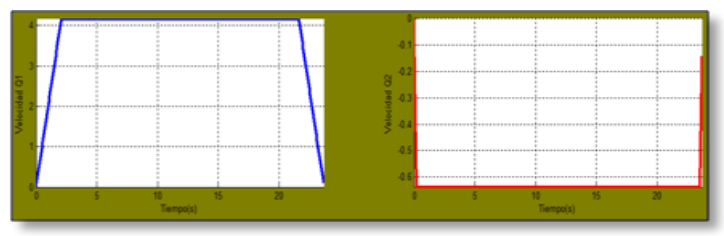

Fig. 6. Comportamiento la velocidad de la articulación $Q_{1}$ y $Q_{2}$.

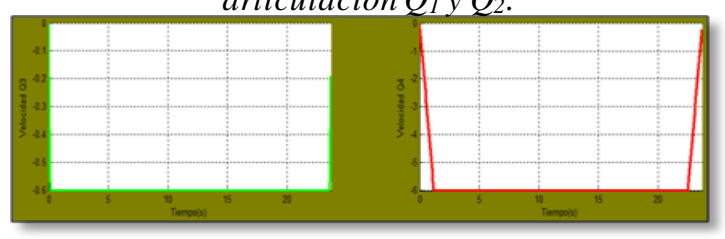

Fig. 7. Comportamiento la velocidad de la articulación $Q_{3}$ y $Q_{4}$. 


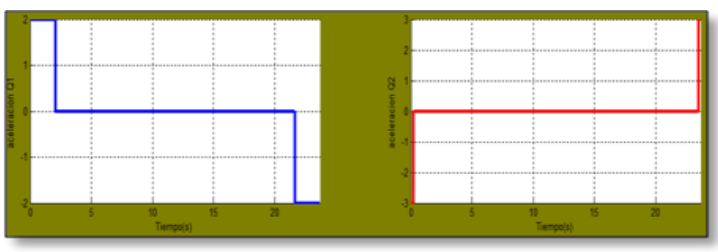

Fig. 8. Comportamiento la aceleración de la articulación $Q_{1}$ y $Q_{2}$.

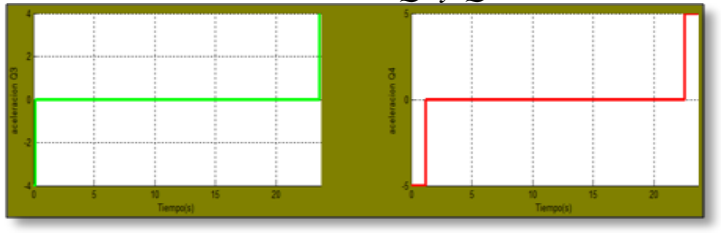

Fig. 9. Comportamiento la aceleración de la articulación $Q_{3}$ y $Q_{4}$.

\section{CONTROL DINÁMICO}

Para el robot cilíndrico o manipulador de 4GDL se aplicó el control prealimentado + realimentado teniendo anteriormente del control cinemático la trayectoria seleccionada se elaboró en simulink una herramienta de software Matlab, las figuras 10 es la representación del control prealimentado lazo abierto, realimentado con lazo cerrado y la $q d$ ( $\mathrm{q}$ deseada) posteriormente de aplicarle el control dinámico, lo seleccionado anteriormente tiene como respuesta una señal más completa y estable al aplicarle perturbaciones los dos tipos de lazos que lo componen cada uno hace su tarea se complementan las falencias del otro, así hay una buena compensación, la figura 11 muestra las trayectorias de cada articulación seguidas por el manipulador aplicando el control anteriormente mencionado obteniendo una respuesta permanente $\mathrm{y}$ sin perturbaciones.

\subsection{Control prealimentado + realimentado}

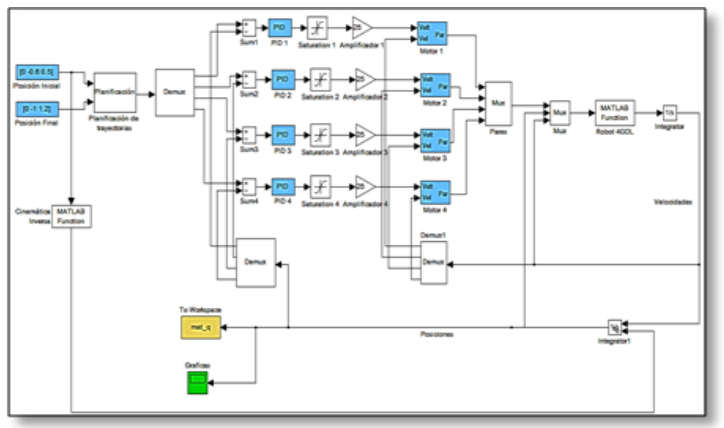

Fig. 10. Control realimentado + prealimentado con par de perturbación con magnitud 5. Este control se implementó con dos lazos uno abierto del control prealimentado y otro realimentado con lazo cerrado de todas las articulaciones.

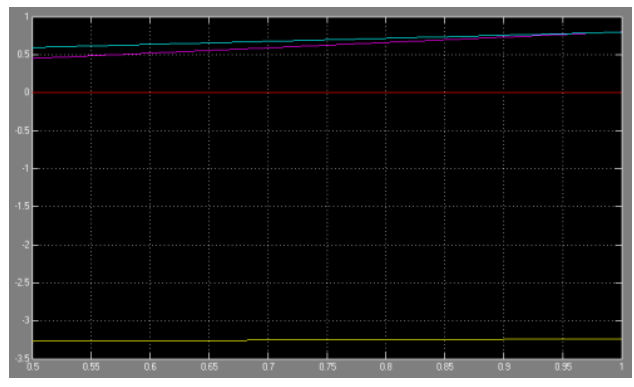

Fig. 11. Gráfica de las trayectorias de cada articulación.

\section{CONCLUS IONES}

Se concluye que para la realización del control cinemático necesariamente se debe tener el modelo cinemático y dinámico del manipulador a diseñar ya que estos son los parámetros de entrada para elaborar el análisis metodológico de este diseño.

El control cinemático tiene en cuenta el punto final y punto inicial o referencia del manipulador serial industrial que al realizar un correcto cálculo de este hace que haya un menor movimiento de los actuadores al momento de realizar la trayectoria programada por el desarrollador aplicando la metodología propuesta.

Se concluye que la trayectoria más óptima para un manipulador serial industrial es la trayectoria coordinada o isócrona ya que todas las articulaciones se mueven simultáneamente se emplea menor tiempo posible y evita exigencias inútiles de velocidad y aceleración a los actuadores de cada articulación.

Se determina que el mejor interpolador para análisis de la trayectoria escogida por el desarrollador es el trapezoidal, ya que evita que la velocidad varié durante la mayor parte del tiempo al momento de cumplir dicha trayectoria.

Se concluye que el óptimo control para aplicar a los actuadores de cada articulación es el control prealimentado más realimentado ya que la unión de estos dos pueden detectar fallos y corrige con mayor rapidez las perturbaciones externas para que el manipulador cumpla la trayectoria real con respecto a la trayectoria propuesta en el control cinemático. Esto se comprueba con las gráficas en la cuales se habla de los controles prealimentado, realimentado y prealimentado más realimentado y se aprecian los resultados de cada control implementado. 


\section{REFERENCIAS}

Hauser K, (2014). "The minimum constraint removal problem with three robotics applications", The International Journal of Robotics Research, Estados Unidos.

Moreno H. A. (2012), Roque Saltaren, Isela Carrera, Lisandro Puglisi, Rafael Aracil. Índices de Desempeño de Robots Manipuladores: una revisión del Estado del Arte, España.

Cieza A. O. B. (2011). Implementacion de una estacion de trabajo mediante un robot serial de 3 grados de libertad para el CETAM, Pontificia Universidad Católica del Peru, Lima Agosto.

Navarro N. N. P., (2011). Modelado cinemático y dinámico de un manipulador de 5 grados de libertad articulado verticalmente, Pontificia Universidad Catolica del Peru, Lima Marzo.
Martínez E. Peña C. Yime E. (2010). Diseño Óptimo de un robot paralelo con configuración delta para aplicación es educativa, Universidad de pamplona y Universidad Tecnológica de Bolívar, Colombia.

Carlos Humberto Esparza, Sergio Omar Carvajal, Víctor Hugo García, (2010). Evaluación de un Sistema de control para un brazo robótico de cinco grados de libertad utilizando una red can-bus, Unidades Tecnológicas de Santander.

Barrientos A. Peñin L. Balaguer C. Aracil R. (2007). Fundamentos de robotica , Mc Graw Hill, Segunda edicioón.

Sabater N. J. M. Martinez V. J. (2012). Guia docente para el diseño de robots de servicio, AIDICO Instituto Tecnológico de la Construcción, España.

Pertuz Ocampo Jairo,(2013). Curso de robotica avanzada, Universidad Nacional Abierta, Colombia. 